\title{
Straightforward Strategies for the Preparation of NH-Sulfoximines: A Serendipitous Story
}

\author{
J. A. Bull*,a \\ Leonardo Degennaro ${ }^{b}$ \\ R. Luisi*,b \\ a Department of Chemistry, Imperial College London, South \\ Kensington, London SW7 2AZ, United Kingdom. \\ ${ }^{\mathrm{b}}$ Department of Pharmacy - Drug Sciences, University of Bari \\ "A. Moro" Via E. Orabona 4, Bari 70125, Italy. \\ * indicates the main/corresponding author. \\ renzo.luisi@uniba.it \\ j.bull@imperial.ac.uk \\ Click here to insert a dedication
}

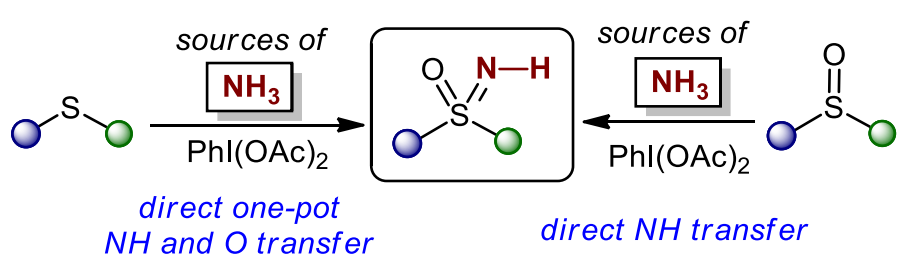

$\mathrm{O}=$ alkyl, aryl, vinyl, cyclic and cyclic

Received:
Accepted:
Published online:
DOI:
Abstract Sulfoximines are emerging as valuable new isosteres for use in medicinal
chemistry, with the potential to modulate physicochemical properties. Recent
developments in synthetic strategy have made the unprotected 'free' NH-
sulfoximine group more readily available, facilitating further study. This account
reviews approaches to NH-sulfoximines, with a focus on our contribution to the
field. Starting from the development of catalytic strategies involving transition
metals, more sustainable metal-free processes has been discovered. In
particular, the use of hypervalent iodine reagents to mediate NH transfer to
sulfoxides is described, along with assessment of the substrate scope.
Furthermore, a one pot strategy to convert sulfides directly to NH-sulfoximines
is discussed, with $\mathrm{N}$ and O transfer occurring under the reaction conditions.
Mechanistic evidence for the new procedures is included as well as relevant
synthetic applications that further exemplify the potential of these approaches.
1. Introduction
2. Strategies to form NH-sulfoximines involving transition metal catalysts
3. Metal-free strategies to prepare NH-sulfoximines
4. Mechanistic evidence for the direct synthesis of NH-sulfoximines from
sulfoxides and sulfides.
5. Further applications
6. Conclusions
Key words

\section{Introduction}

Since the discovery of the first sulfoximine in 1949,1 the chemistry of sulfoximines has developed from a rather niche area to a field with wide and expanding applications. ${ }^{2-7}$ The sulfoximine group is chemically stable, including stable to hydrolysis, and has stable configuration. As such uses of the sulfoximine functional group encompass chiral auxiliaries, ${ }^{3}$ ligands in asymmetric catalysis, ${ }^{4}$ and directing groups for $\mathrm{C}-\mathrm{H}$ functionalisation, ${ }^{5}$ as well as agrochemicals $^{6}$ and medicinal chemistry. ${ }^{7}$ Much of the development over the last decade has been facilitated by the introduction of new and more practical synthetic methodologies. ${ }^{8,9,10}$ These advances in the field, brought about an increasing interest in sulfoximines in life sciences and drug discovery. ${ }^{11-14}$ In Figure 1 are reported some biologically relevant molecules bearing the sulfoximine moiety.
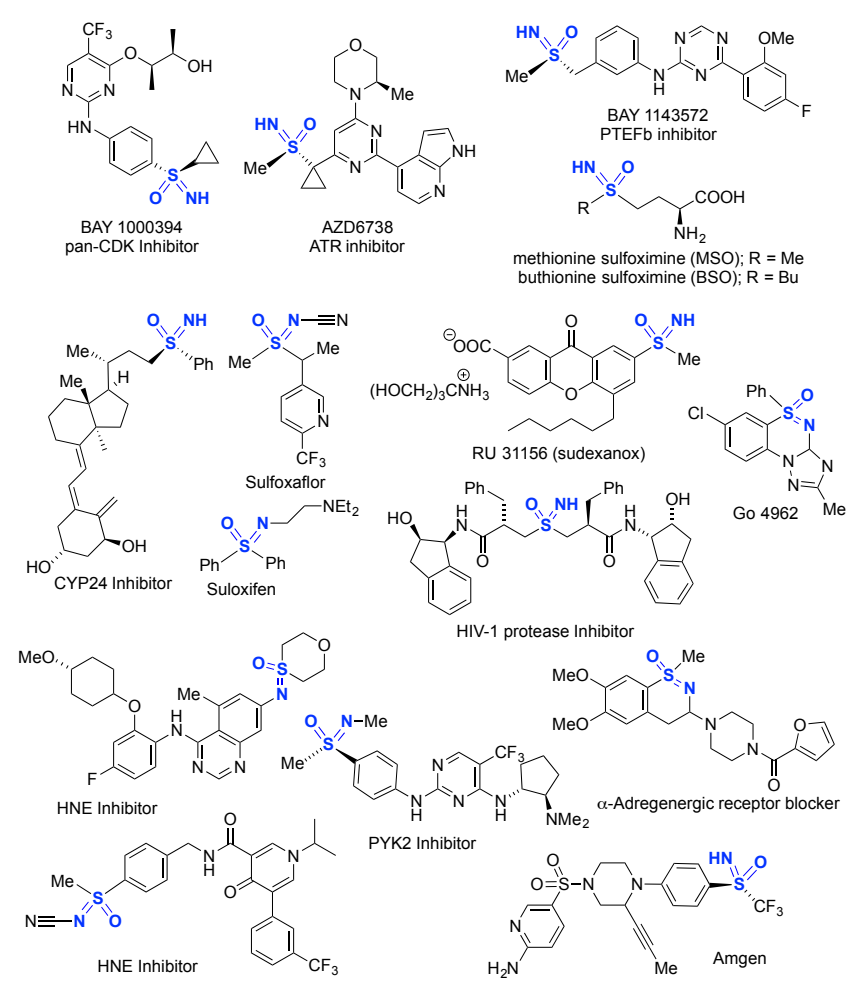

Figure 1. Examples of biological relevant sulfoximine compounds.

Our understanding of the potential of the sulfoximine group in drug discovery is growing. Compounds containing $\mathrm{NH}$ sulfoximines from Bayer (BAY1000394 and BAY1143572) and AstraZeneca (AZD6738) are paving the way in current clinical trials. ${ }^{12}$ While there remain relatively few published studies on sulfoximine properties relevant to medicinal chemistry, high metabolic stability, hydrogen-bond acceptor/donor capabilities and desirable physicochemical properties are observed.7,13,14 As isosteres for sulfones, the sulfoximine group often provides improved solubility, and also presents additional complexity with an additional attachment point. In a recent study, Bolm and co-workers from Boehringer Ingelheim reported the analysis of a series of matched 
molecular pairs to compare sulfoximines against other sulfur functionality on various simple substrates. ${ }^{13}$ Sirvent and Lücking from Bayer evaluated the sulfoximine functionality as isosteres for amines by incorporation into analogues of marketed drugs and advanced clinical candidates with favourable comparison of physical and biological properties. ${ }^{14}$

From $\mathrm{NH}$-sulfoximines, the $\mathrm{N}$-atom offers potential to introduce further molecular diversity through an increasingly broad array of transformations, as exemplified by $\mathrm{N}$-trifluoromethylation, 15 arylation, ${ }^{16} \quad$ aroylation, ${ }^{17} \quad$ alkynylation, ${ }^{18}$ alkylation, ${ }^{19}$ propargylation, ${ }^{20}$ cyanation, ${ }^{21}$ thioetherification, ${ }^{22,23}$ and intramolecular halocyclization reactions. ${ }^{24}$ Racemic NH-sulfoximines can also be subjected to catalytic kinetic resolution. ${ }^{25}$

As a consequence of the importance of the sulfoximine functionality, the development of strategies for the synthesis and incorporation of this structural motif is still a vigorous research area. To access NH-sulfoximines, different synthetic approaches are now available (Scheme 1). Either the oxygen or the nitrogen group may be introduced first, affording the corresponding sulfoxide or sulfilimine ${ }^{26}$ respectively, using electrophilic reagents. Further oxidation of sulfilimines or imination of sulfoxides, affords the sulfoximine. Commonly a protected $\mathrm{N}$-group is involved, meaning 3-step sequences are required to access the free $\mathrm{NH}$ sulfoximine from sulfides.

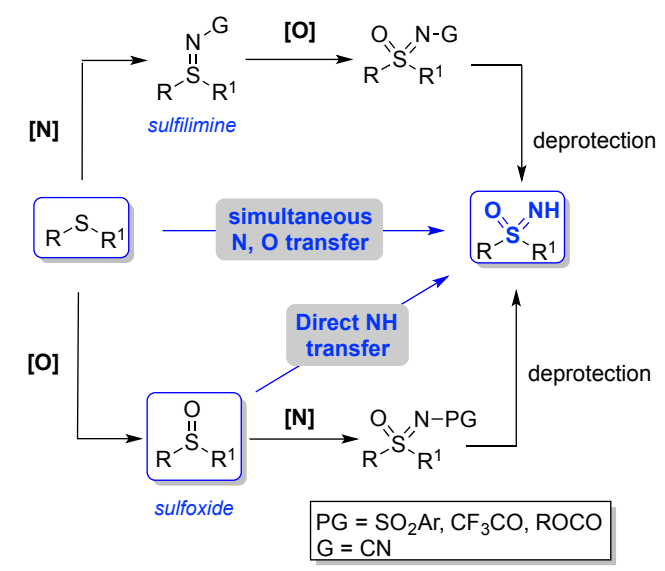

Scheme 1. Strategies for accessing $\mathrm{NH}$-sulfoximines.

In this account, we review methods for the synthesis of $\mathrm{NH}$ sulfoximines, through transition metal catalyzed and metal free methods. In 2015, Bolm produced a comprehensive review on methods for sulfoximine synthesis. ${ }^{8 a}$ Here, we aim to highlight important advances for the preparation of $\mathrm{NH}$ sulfoximines since 2015, with a focus on our own contributions. In particular, we recently reported straightforward strategies for the preparation of $\mathrm{NH}$-sulfoximines by deprotection of easily removable $\mathrm{N}$-protecting groups, the direct introduction of the $\mathrm{NH}$ group on sulfoxides, and the simultaneous transfer of $\mathrm{O}$ and $\mathrm{NH}$ groups on sulfides. We wish to report our perspective on the progress of the work, how our strategy complements other available procedures, and underlying mechanistic investigations.

\section{Strategies to form NH-sulfoximines involving transition metal catalysts}

When we first entered the field in 2015, there were several powerful methods available for transition metal-catalyzed $\mathrm{N}$ - transfer to sulfoxides, being the most common route to protected sulfoximines (Scheme 2).8a The use of various transition-metal catalysts allows the generation and stabilization of metal nitrenoid species responsible for the $\mathrm{N}$-transfer to the sulfur atom. The use of $\mathrm{Cu}, \mathrm{Rh}, \mathrm{Ag}$ and $\mathrm{Fe}$ catalysis has been employed for the transfer of sulfonamide groups by activated nitrogen species (e.g. $\mathrm{PhI}=\mathrm{NSO}_{2} \mathrm{Ar}$, Scheme 2,a).27,28 Bolm reported the use of a combination of trifluoroacetamide and $\mathrm{PhI}(\mathrm{OAc})_{2}$ in the presence of $\mathrm{Rh}_{2}(\mathrm{OAc})_{4}$ catalyst for transferring the $\mathrm{N}-\mathrm{COCF}_{3}$ group to sulfoxides (Scheme 2,b). ${ }^{28}$ The same author developed the $\mathrm{N}$-acyl transfer to sulfoxides using 1,4,2-dioxazol-5-ones under $\mathrm{Ru}$ catalyzed photochemical conditions (Scheme 2,c). ${ }^{29}$ With these methods, removal of the $\mathrm{N}$-substituent could give access to free $\mathrm{NH}$ sulfoximines. While $\mathrm{N}$-tosyl groups are quite challenging to remove, it has been demonstrated that the nosyl (Ns) group could be removed by treatment with thiophenol and cesium carbonate (Scheme 3,a). ${ }^{26 \mathrm{~b}}$ On the other hand, $\mathrm{N}$-trifluoroacetyl sulfoximines can be effectively $N$-deprotected by treatment with $\mathrm{K}_{2} \mathrm{CO}_{3} / \mathrm{MeOH}$ (Scheme 3,b). ${ }^{28}$

a)

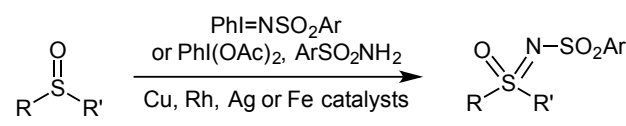

b)

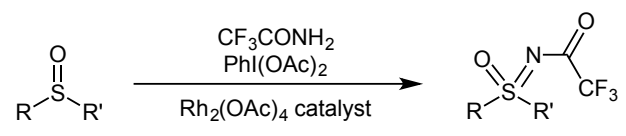

c)

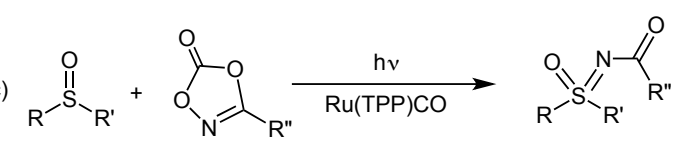

d)

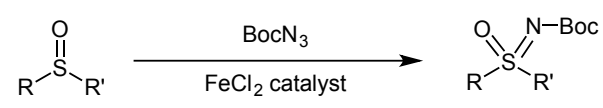

Scheme 2. Metal-based approaches to N-protected sulfoximines

a)

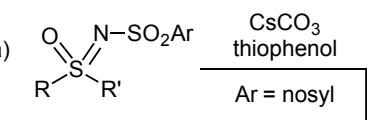

b)

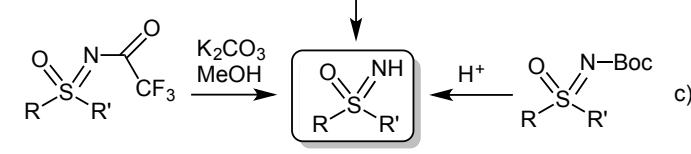

d)

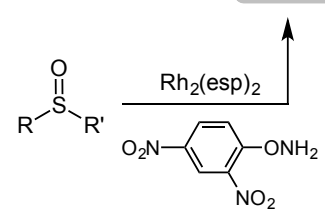

Scheme 3. Strategies for accessing NH-sulfoximines.

To date, there is only one direct transition metal-catalyzed method for the preparation NH sulfoximines. Richards reported a direct Rhcatalyzed method for $\mathrm{NH}$ transfer to sulfoxides using 0-(2,4dinitrophenyl)-hydroxyl-amine as nitrogen source, and $\mathrm{Rh}_{2}(\mathrm{esp})_{2}$ as the catalyst (Scheme $3, \mathrm{~d}) .^{30}$

For the purposes of our initial investigations, we were interested in carbamate protected sulfoximines. We envisioned that these would be stable to allow synthetic transformations prior revealing the $\mathrm{NH}$-sulfoximine, and would be straightforward to deprotect under well understood conditions. In particular, sulfoximine carbamates bearing $\mathrm{N}-\mathrm{Boc}$ and $\mathrm{N}-\mathrm{Cbz}$ groups would present orthogonal 
$\mathrm{N}$-deprotection strategies. However, we were surprised that similar catalytic methods to form carbamate protected sulfoximines from carbamates were not available. Indeed, carbamate protecting groups were commonly installed through reaction of $\mathrm{NH}$ sulfoximines formed by other methods. ${ }^{12 a}$ The only direct method, developed by Bach, used $\mathrm{FeCl}_{2}$-catalyzed transfer of the N-Boc group to the sulfur atom of sulfoxides, using potentially explosive azides as the nitrogen source (Scheme 2,d). ${ }^{31}$ Bach demonstrated the removal of the Boc group in acid conditions, using TFA (Scheme 3, c)

With the aim of providing new $N$-protected sulfoximines, we developed a Rh-catalyzed nitrogen transfer from tert-butyl, benzyl, methyl, ethyl, phenyl and allyl carbamates (Scheme 4). ${ }^{32}$ We began our study exploring the use of $\mathrm{BocNH}_{2}$ and $\mathrm{PhI}(\mathrm{OAc})_{2}$, in the presence of $\mathrm{Rh}_{2}(\mathrm{OAc})_{4}$ in order to generate an activated nitrenoid species (i.e. BocN=Rh) for the $\mathrm{N}$-transfer to methyl tolylsulfoxide 1a. Adapting Bolm's protocol, ${ }^{28}$ using rhodium catalysis and magnesium oxide as base, ${ }^{33}$ we were delighted to observe the direct formation of the N-Boc sulfoximine 2a in very good yield when running the reaction at $40{ }^{\circ} \mathrm{C}$. It is worth pointing out that the reaction required the metal catalysis, no reaction occurred in the absence of $\mathrm{Rh}_{2}(\mathrm{OAc})_{4}$, and the reaction was performed under air without requiring anhydrous dichloromethane.

The scope of this reaction was widely explored, with examples in Scheme 4. Several N-Boc sulfoximines 2a-i were prepared in good to excellent yields. Under optimized reaction conditions, it was also possible the transfer of methyl and ethyl carbamates to the sulfur atom of sulfoxides obtaining sulfoximines 3a-d and 4a-c respectively. In the case of four-membered thietane 1-oxide, $5 \mathrm{~mol} \%$ of Rh-catalyst was needed for the $\mathrm{N}$-transfer of the carbamates leading to thietane sulfoximines $\mathbf{2 i}$, 3d and $\mathbf{4 c}$ (Scheme 4). The stereospecificity of the transfer of the $N$-Boc group to the sulfur was demonstrated by performing the reaction on enantioenriched (S)-1a (er 97:3). The reaction occurred with complete retention of configuration, and preserving the er in $\mathrm{N}$ Boc-sulfoximine 2a. ${ }^{32,34}$

Slightly different reaction conditions were needed for the transfer of benzyl, phenyl and allyl carbamates, which may undergo unfavorable coordination between the $\pi$-system in carbamate substituents and the catalyst. These substrates were less reactive, but this could be somewhat ameliorated by running the reaction at an increased $0.3 \mathrm{M}$ concentration in sulfoxide. Under these optimized conditions, N-benzyloxycarbonyl sulfoximines 5a-c, $\mathrm{N}$ phenyloxycarbonyl sulfoximines $\mathbf{6 a}, \mathbf{b}$ and $\mathrm{N}$-allyloxycarbonyl sulfoximines $\mathbf{7 a}$, b could be prepared in acceptable yields (Scheme 4).

The usefulness of $N$-protected sulfoximines was demonstrated by further manipulations: $N$-Boc and $N$-Cbz sulfoximines $\mathbf{2 b}$ and $\mathbf{5 b}$ underwent smooth $\mathrm{Pd}$ - and Fe-catalyzed cross coupling reactions. ${ }^{32}$ The possibility to generate free $\mathrm{NH}$-sulfoximines was demonstrated with the deprotection of $\mathrm{N}$-Cbz- and $\mathrm{N}$-Bocsulfoximines 2a, 5a and 2i (Scheme 5). By using standard acidic conditions, the Boc group was removed from N-Boc sulfoximines $\mathbf{2 a}$ and $\mathbf{2} \mathbf{i}$ in good to excellent yields, obtaining the corresponding $\mathrm{NH}$-sulfoximines 8a and $\mathbf{9}$ respectively. By using hydrogenolysis conditions (Scheme 5), from $\mathrm{N}-\mathrm{Cbz}$ sulfoximine $\mathbf{5 a}$, the corresponding $\mathrm{NH}$-sulfoximine $\mathbf{8 a}$ was obtained in $77 \%$ yield.

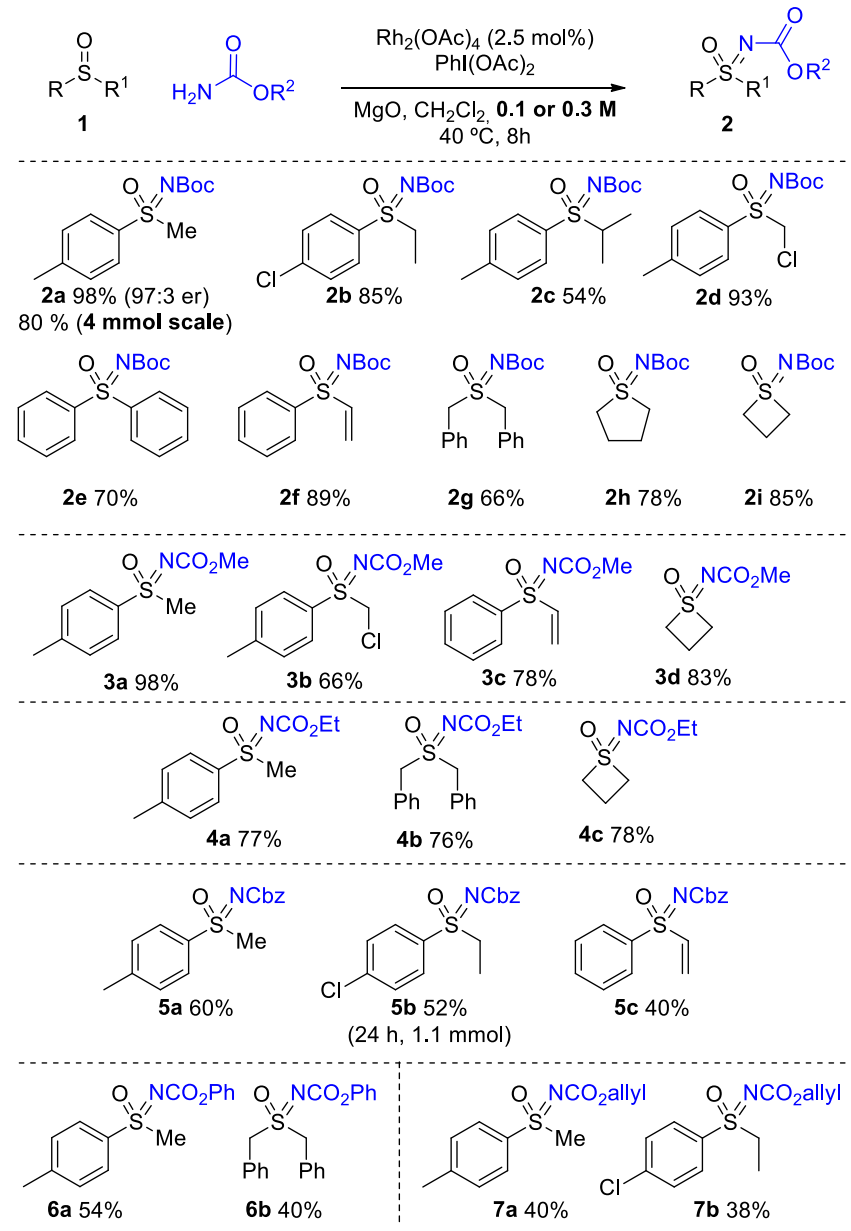

Scheme 4

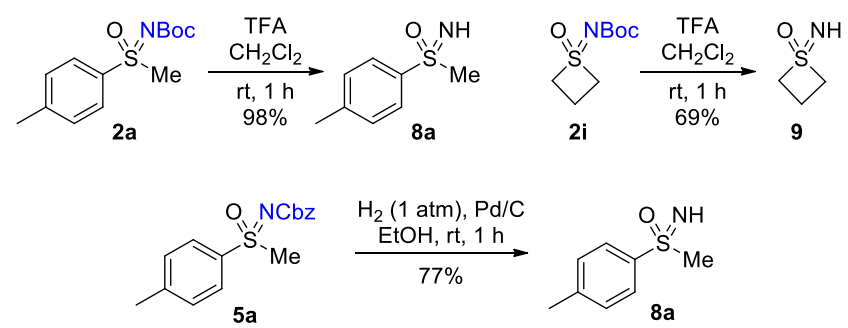

Scheme 5

This synthetic strategy for the preparation of $N$-protected sulfoximines, was effectively employed for the preparation of the target molecule MSO (methionine sulfoximine).32,35 N-Boc sulfoximine 11, formed from methionine sulfoxide $\mathbf{1 0}$ (a 1:1 mixture of diastereoisomers) by Rh-catalyzed N-Boc transfer, was fully deprotected under acidic conditions affording MSO in 54\% yield over the two steps (Scheme 6).

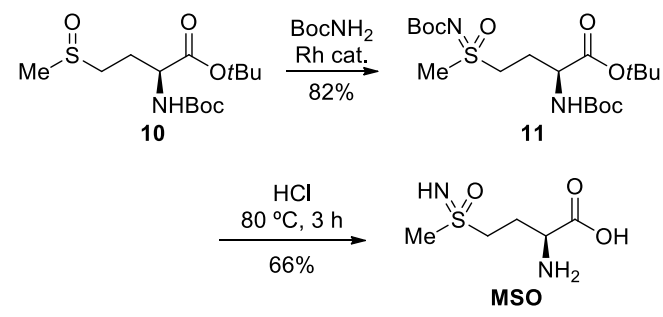

Scheme 6 
This carbamate transfer protocol appears to be a general and convenient procedure for the preparation of sulfoximine carbamates. Our approach complements well with other already available strategies to $\mathrm{N}$-protected sulfoximines, and may facilitate synthetic planning by allowing flexible removal of protecting groups to access $\mathrm{NH}$ sulfoximines.

\section{Metal-free strategies to prepare $\mathrm{NH}$-sulfoximines}

The use of transition metal catalysts such as rhodium can be expensive on large scale, and in the late-stage preparation of pharmaceutical products presents toxicological issues. In order to render the preparation of sulfoximines more sustainable, several transition metal-free strategies have been invented (Scheme 7). For the direct preparation of $\mathrm{NH}$-sulfoximines from sulfoxides, one of the oldest but still used procedures relies on the use of $\mathrm{NaN}_{3}$ under acidic conditions (Scheme 7,a). ${ }^{36}$ However, the harsh reaction conditions and the hazards related to the development of $\mathrm{HN}_{3}$, makes this strategy unattractive. Interestingly, the flow technology has been suggested as a possible solution for a safer use of azides. $9 \mathrm{~b}$ However, even under flow conditions, this strategy was not compatible with enantioenriched starting materials. The use of the Eaton's reagent (phosphorus pentoxide, in methanesulfonic acid) and $\mathrm{NaN}_{3}$ has recently been proposed by Liang for accessing $\mathrm{NH}_{-}$ sulfoximines. ${ }^{37}$ Another old strategy for the preparation of NHsulfoximines uses 0-mesitylene sulfonylhydroxylamine (MSH) for the direct transfer of the $\mathrm{NH}$ group to sulfoxide (Scheme 7, b). However, MSH is an unstable reagent that need to be prepared before use. ${ }^{38}$

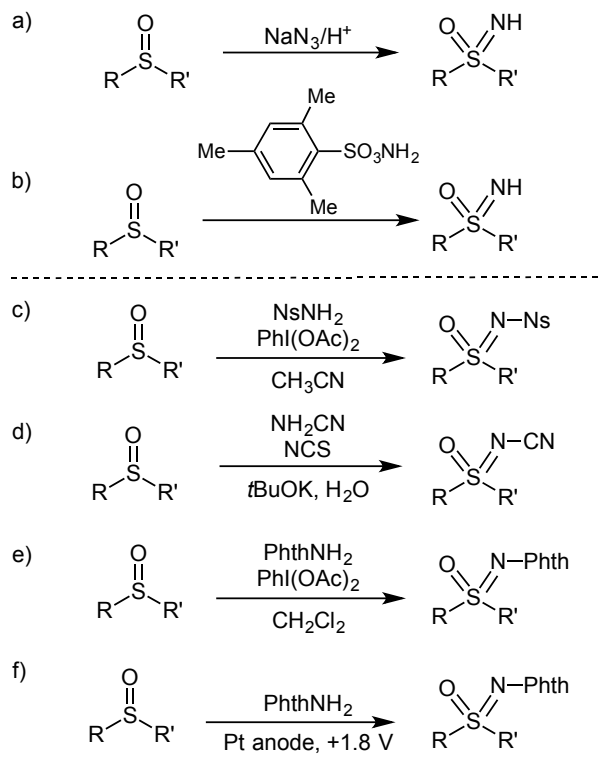

Scheme 7

Several alternative milder metal-free iminations of sulfoxides have been developed leading to N-protected sulfoximines (Scheme 7). In one of the earlier reports, Bolm developed a metal-free transfer of the $p$-nitro-benzenesulfonylamide $\left(\mathrm{NsNH}_{2}\right)$ to the sulfur of sulfoxides in the presence of $\mathrm{PhI}(\mathrm{OAc})_{2}$ as the oxidant (Scheme 7,c). ${ }^{39}$ Very recently, Bolm reported a metal-free synthesis of $\mathrm{N}$ cyano sulfoximines by $\mathrm{N}$-transfer from cyanamide $\left(\mathrm{NCNH}_{2}\right)$ in presence of $\mathrm{N}$-chlorosuccinimide (NCS) as the oxidant and potassium tert-butoxide as the base (Scheme 7,d).40 Yudin introduced two metal-free methodologies, leading to $\mathrm{N}$ phthalimido sulfoximines,. One simple protocol, relies on the use of $\mathrm{N}$-amino phthalimide (Phth- $\mathrm{NH}_{2}$ ) in the presence of $\mathrm{PhI}(\mathrm{OAc})_{2}$ as the oxidant in dichloromethane (Scheme 7,e). ${ }^{41}$ Yudin also developed a "green" and mild electrochemical sulfoxide imination using Phth- $\mathrm{NH}_{2}$ as the iminating agent (Scheme 7,f). ${ }^{42}$

Removal of these N-protecting groups has been demonstrated in isolated examples. Deprotecting the $\mathrm{CN}$ group needed two additional steps to reveal the $\mathrm{NH}$ sulfoximine consisting of treatment with $\mathrm{Tf}_{2} \mathrm{O}$ followed by basic hydrolysis (Scheme 8,a). ${ }^{43} \mathrm{~A}$ direct removal of the cyano group is also possible under rather forcing conditions: $50 \%$ aqueous sulfuric acid at $110{ }^{\circ} \mathrm{C} .44$ Yudin proved that the $\mathrm{N}$-phthalimido group could be removed electrochemically using a Pt cathode in the presence of a methanolic solution of an ammonium salt (Scheme 8,b). ${ }^{42}$

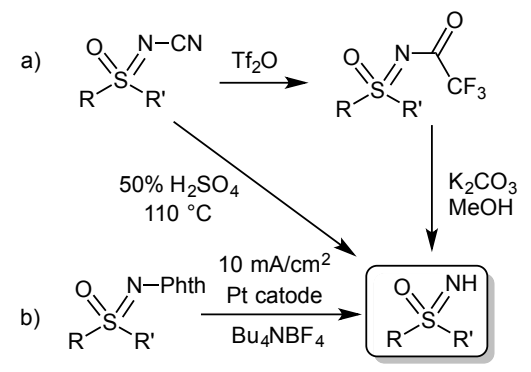

Scheme 8

We considered that development of a safe, metal-free and direct method for the NH-transfer to sulfoxides, particularly using convenient inexpensive nitrogen sources, would offer considerable value. Building on our previous work on the Rh-catalyzed transfer of alkyl carbamates to sulfoxides (Scheme 4), we explored the possibility of using a carbamic salt, as the $\mathrm{N}$-source, expecting the $\mathrm{NH}$-sulfoximine to be formed directly due to the driving force for decarboxylation to occur, either before or after the $\mathrm{N}$-transfer.

Ammonium carbamate was chosen as an inexpensive and easily handled nitrogen source, and tested in metal catalyzed (Rh and Fe) as well as metal-free $\mathrm{N}$-transfer reactions. To our delight, we were able to obtain the desired NH-sulfoximine.45 However, unexpectedly and serendipitously, we found that the reaction proceeded efficiently without requiring a transition metal catalyst. This metal free protocol, opened up new perspectives in the field of the direct preparation of $\mathrm{NH}$-sulfoximines as well as in $\mathrm{N}$-transfer methodologies. The reaction was optimized using sulfoxide $\mathbf{1 a}$, and could be conducted under several reaction conditions (Scheme 9)

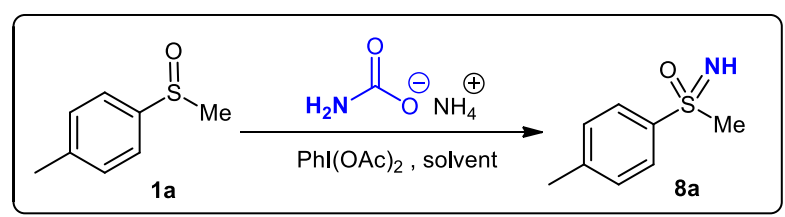

\section{Conditions}

Yield

a) Toluene, $\mathrm{H}_{2} \mathrm{NCO}_{2} \mathrm{NH}_{4}$ (2 equiv) Phl(OAc) 2 (2 equiv), $\mathrm{MgO}, 35^{\circ} \mathrm{C}, 16 \mathrm{~h} 90 \%$ b) $\mathrm{MeCN}, \mathrm{H}_{2} \mathrm{NCO}_{2} \mathrm{NH}_{4}$ (2.5 equiv), $\mathrm{Phl}(\mathrm{OAc})_{2}\left(1.5\right.$ equiv), $25^{\circ} \mathrm{C}, 3 \mathrm{~h} \quad 90 \%$ c) $\mathrm{MeOH}, \mathrm{H}_{2} \mathrm{NCO}_{2} \mathrm{NH}_{4}$ (4 equiv), $\mathrm{Phl}(\mathrm{OAc})_{2}$ (3 equiv), $25^{\circ} \mathrm{C}, 30 \mathrm{~min} \quad 96 \%$ Conditions c), $10 \mathrm{mmol}$ scale $91 \%$ 
Indeed, the reaction could be run in toluene, acetonitrile and methanol. Using toluene as the solvent (conditions a, Scheme 9), mixing ammonium carbamate with bisacetoxyiodobenzene and magnesium oxide at $35{ }^{\circ} \mathrm{C}$ for $16 \mathrm{~h}$, led to sulfoximine 8a with $90 \%$ yield. The heterogeneous nature of the reaction in toluene affected the rate of dissolution of ammonium carbamate, therefore to reduce the reaction time more polar solvents were employed. Acetonitrile and methanol were both successful, providing full conversion in the absence of the base (conditions b,c, Scheme 9). However, higher yields (96\%) and shorter reaction time (30 min) were achieved in methanol (conditions c, Scheme 9). This protocol is very simple and easy to perform in an open flask, and scalable as demonstrated by performing the reaction on a $10 \mathrm{mmol}$ scale (1a, $91 \%$ yield $\mathbf{8 a}$ ). To highlight the practical nature of this method, we prepared a video of the protocol. ${ }^{46}$

The scope of the reaction has been widely investigated mainly using $\mathrm{MeOH}$ as the solvent (Scheme 10).45 Different protocols and solvents can also be used depending on the solubility of the starting sulfoxide.
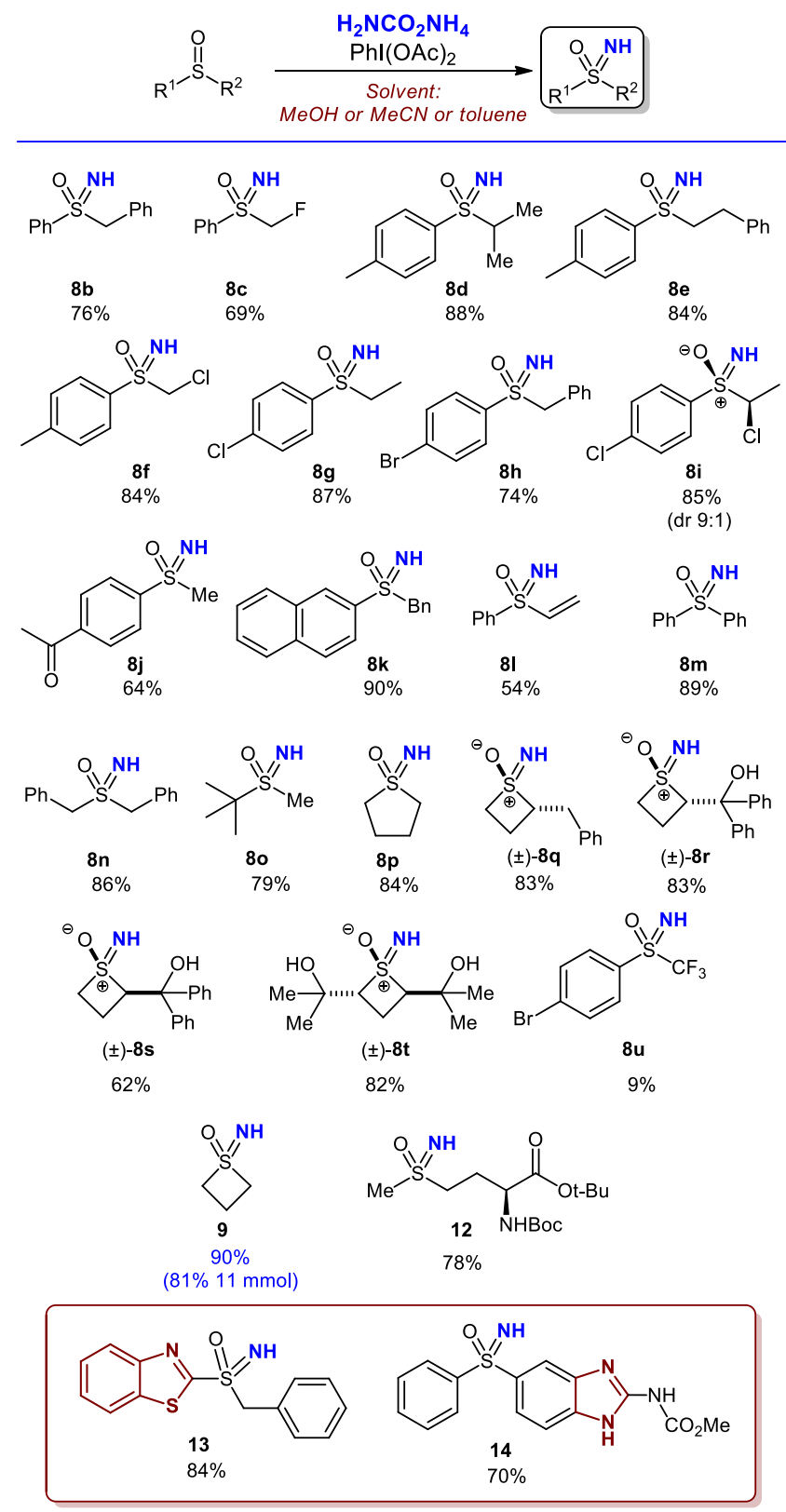

\section{Scheme 10}

As reported in Scheme 10,45 the NH-transfer occured on various sulfoxides leading to the corresponding NH-sulfoximines 8b-t in good to excellent yields. We found the protocol compatible with aryl and alkyl substituted sulfoxides (e.g. 8b-o), as well as cyclic sulfoxides as in the cases of $\mathbf{8 p - t}$. A lower yield was observed with phenylvinylsulfoxide, leading to $\mathbf{8 1}$ in $54 \%$ yield. A trifluoromethylsulfoxide provided $\mathbf{8 u}$ in only $9 \%$ yield due to the lower nucleophilicity of the sulfoxide. Concerning the stereochemical outcome of the NH-transfer methodology, complete stereoselectivity was observed in sulfoximines 8q-t (Scheme 10) deriving from diastereomerically pure cyclic sulfoxides. Similarly, the 9:1 diastereomeric ratio of a $\alpha$-chlorinated sulfoxide was retained in sulfoximine $\mathbf{8 i}$ (Scheme 10). The reaction on enantioenriched sulfoxides resulted in enantioenriched sulfoximines $(S)-\mathbf{8 a},(R)-\mathbf{8 b}$ and $(R)-\mathbf{8 h}$ (er>97:3), with complete retention of configuration from the starting materials. ${ }^{45}$ The scalability of the procedure was also demonstrated with thietanesulfoximine $\mathbf{9}$, prepared on an $11 \mathrm{mmol}$ scale in $81 \%$ yield.

Functional group compatibility of this NH-transfer to form sulfoximines is important to ensure synthetic efficiency and in later stage applications. In particular, for applications in medicinal chemistry, compatibility with heterocycles is crucial. The conditions were shown to be tolerant of various functional groups in the sulfoxides, including free hydroxyl groups in sulfoximines 8r-t (Scheme 10). Furthermore, protected methionine sulfoxide smoothly undergoes $\mathrm{NH}$-transfer providing protected MSO derivative $\mathbf{1 2}$ in good yields (Scheme 10). To give an indication of the tolerance of the reaction to a much wider array of possible sulfoxide substrates, the procedure was subjected to Glorius' robustness screen. ${ }^{45,47}$ The standard reaction with sulfoxide 1a, was performed in the presence of stoichiometric quantities of various functionalised additives (Figure 2). The majority of the heterocycles tested were found to be compatible, giving the product 8a in yields in the range $85-95 \%$. Importantly, heterocycles with basic nitrogen groups were found compatible with the reaction. Very high yields were observed in the presence of pyridine, pyrimidine, quinoline, imidazole, thiazole, benzoxazole and benzothiazole. According to this robustness screen, sulfoxides bearing benzothiazole and imidazole groups were expected to provide very good yields of the corresponding sulfoximines. Therefore, sulfoximine 13, bearing the benzothiazole substituent, was targeted, and successfully prepared in $84 \%$ yields (Scheme 10). Similarly, oxfendazole, an anthelmintic containing benzimidazole, was shown to undergo NH-transfer furnishing the corresponding sulfoximine 14 in 70\% (Scheme 10).

On the other hand, electron rich heterocycles such as indole, furan and thiophene were less well tolerated, leading to a poor recovery of the added heterocycle. The pyrrole group required $\mathrm{N}$-protection with electron-withdrawing substituents, such as the Boc group, in order to be tolerated in the reaction. Various other functional groups were tested, and the NH-transfer protocol proved to be compatible with alkene, alkyne, alkyl amine, phenol, ester, aldehyde and nitrile functionality. The results in Figure 2 demonstrate a high degree of compatibility of the reaction conditions for a wide range of pharmaceutically relevant functionalities. 


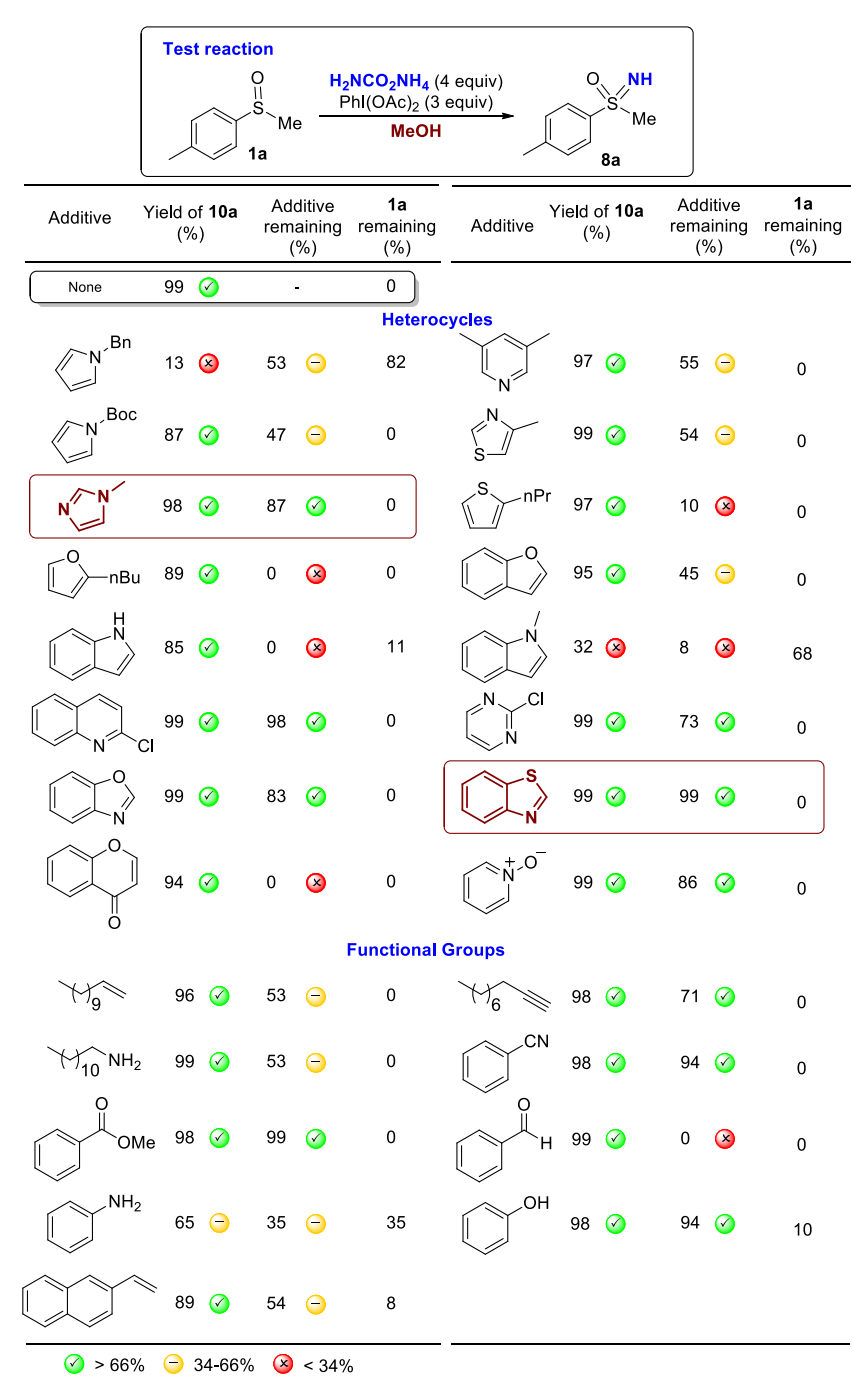

Figure 2. Robustness test

Next, we considered the use of this straightforward direct $\mathrm{NH}$ transfer procedure with sulfides as substrates. ${ }^{48}$ Aiming to develop a method for the direct preparation of $\mathrm{NH}$-sulfilimines, by imination of the sulfur atom, sulfide $\mathbf{1 5 a}$ was reacted under the same reaction conditions employed for the imination of sulfoxides. (Scheme 11). Surprisingly, by using PhI(OAc) ${ }_{2}$ and $\mathrm{NH}_{2} \mathrm{CO}_{2} \mathrm{NH}_{4}$ in methanol, sulfoximine 8a was obtained in very high yield rather than the expected sulfilimine. Considering that most preparations of $\mathrm{NH}$-sulfoximines are based on multi-step syntheses starting from sulfides (Scheme 1), the availability of a selective strategy for the $\mathrm{N}$ - and $\mathrm{O}$ - transfer in the same reaction to generate $\mathrm{NH}$ sulfoximines was extremely attractive, and promised a considerable advance. In preliminary experiments on sulfide 15a, the efficacy of the protocol used for the imination of sulfoxides was proven. The use of ammonium carbamate $\left(\mathrm{NH}_{2} \mathrm{COONH}_{4}\right)$ in the presence of of $\mathrm{PhI}(\mathrm{OAc})_{2}$ (2.5 equiv) at $25^{\circ} \mathrm{C}$, in $\mathrm{MeOH}$, toluene or acetonitrile, led to exclusive formation of the corresponding sulfoximine 8a (Scheme 11).

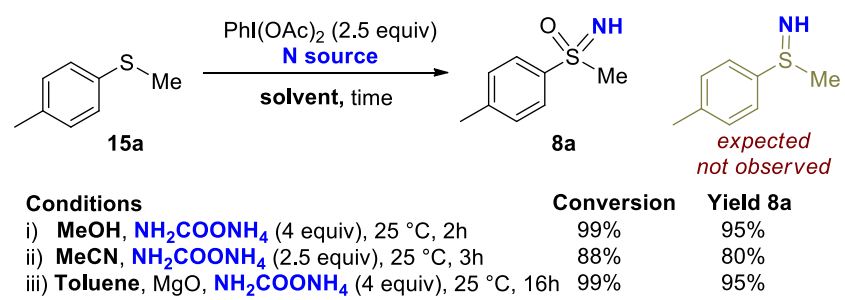

\section{Scheme 11}

This straightforward protocol was tested with several sulfides verifying the robustness of the methodology (Scheme 12).48 The reaction tolerated aryl, alkyl, cycloalkyl, benzyl and alkenyl substituted sulfides (Scheme 12). Various functional groups (OMe, $\mathrm{CF}_{3}$, N-Boc, allyl) and heterocycles were also found compatible with this protocol.<smiles>C=CCS(=O)(=O)c1ccc(OC)cc1</smiles>

Scheme 12

The wide scope and functional groups tolerance makes this strategy useful for the direct introduction of the sulfoximine moiety in organic molecules. Currently, this approach affords racemic sulfoximine products. The direct enantioselective synthesis of sulfoximines from sulfides presents an important future aim.

Mechanistic evidence for the direct synthesis of $\mathrm{NH}$ sulfoximines from sulfoxides and sulfides

The mechanisms of these direct NH transfer methods mediated by hypervalent iodine reagents were intriguing and by no means clear. This prompted us to undertake considerable investigation to provide mechanistic insights. In the NH-transfer to sulfoxides, our approach involved chemical experiments jointly with NMR and HRMS investigation to prove the role of the ammonium carbamate, and the structure of the most likely intermediates. ${ }^{45}$ 
Ultimately the role of ammonium carbamate was shown to simply be a convenient source of ammonia. As reported in Scheme 13, using methanol as the solvent, a fast ligand exchange reaction with $\mathrm{PhI}(\mathrm{OAc})_{2}$ releases acetic acid which decomposes the ammonium carbamate to ammonia and ammonium acetate. ${ }^{49}$ The role of ammonia, as the effective $\mathrm{N}$-source, was further demonstrated by using alternative sources of ammonia such as a solution of ammonia in methanol and ammonium acetate (Scheme 13, b). ${ }^{45}$ In all cases, complete conversion of sulfoxide $\mathbf{1 a}$ to the corresponding sulfoximine $\mathbf{8 a}$ was observed.

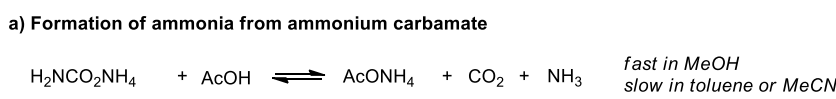

b) Effect of alternative ammonia sources

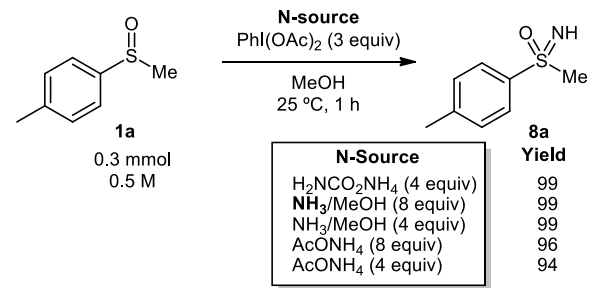

\section{Scheme 13}

The reaction monitored by NMR in deuterated toluene, acetonitrile or methanol led to complete conversion in each case, and a faster reaction in $\mathrm{MeOH}$ or $\mathrm{MeCN}$ over toluene. 45 However, NMR experiments did not provide evidence about likely intermediates; the iodonium salt $\mathbf{1 6}$ was the only detectable species (Scheme 14), which collapsed to the NH-sulfoximine over time. The hypervalent iodonium salt could be also prepared by simply mixing the $\mathrm{NH}-$ sulfoximine with $\mathrm{PhI}(\mathrm{OAc})_{2}$. At this stage, it seemed most likely that the sulfoximine product could be reacting very rapidly with excess $\mathrm{PhI}(\mathrm{OAc})_{2}$ while the reaction is progressing to give the iodonium salts (Scheme 14). However, HRMS studies, indicated this may not be the full story. The iodonium salts were found to be labile when aged for a prolonged time in solution, or under basic work-up conditions giving the corresponding $\mathrm{NH}$-sulfoximine.

Interestingly, similar hypervalent iodonium salts were recently reported by Bolm, and used as sources of sulfoximines for $\mathrm{N}-\mathrm{C}$ bond forming reactions. ${ }^{18 \mathrm{~b}}$ In the Bolm work, the sulfinimidoyl iodonium salts, of general structure 17, precipitated from acetonitrile by mixing a NH-sulfoximine with methoxy(tosyloxy)iodobenzene (Scheme 20, a). Considering this report, and surprised by some spectroscopic discrepancies, we found that sulfinimidoyl iodonium salt 17 could be obtained by mixing the $\mathrm{NH}$-sulfoximine with $\mathrm{PhI}(\mathrm{OAc})_{2}$ in acetonitrile and adding tosic acid. A white solid, corresponding to iodonium salt $\mathbf{1 7}$, suddenly separated from the solution. Running the experiment in deuterated acetonitrile, allowed us to conclude that in solution a fast ligand exchange around the hypervalent iodine occurs, and that the most stable (or insoluble) complex, the tosylate salt, separated as the solid. 50
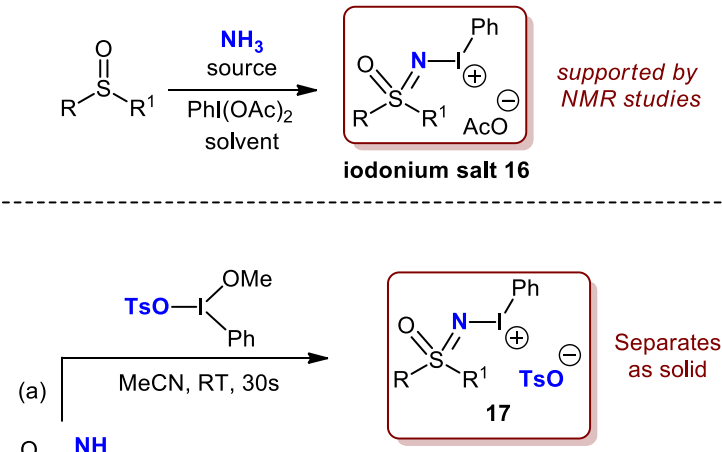

$\mathrm{R}^{-}{ }^{\mathrm{O}} \mathrm{R}^{1}$

(b)

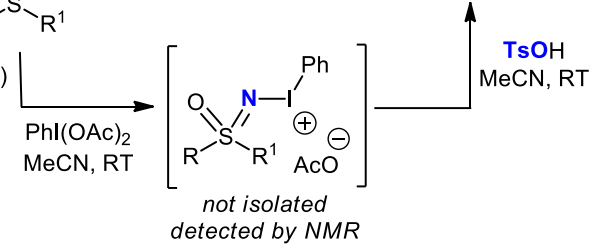

Scheme 14

Flow HRMS analysis provided additional evidences to probe the mechanism of the $\mathrm{NH}$ transfer reaction. ${ }^{45,51}$ In fact, signals consistent with an iminoiodinane $(\mathrm{PhI}=\mathrm{NH})$ as well as an unprecedented iodonitrene $\left(\mathrm{PhI}=\mathrm{N}^{+}\right)$species $^{52}$ were observed after mixing a solution of $\mathrm{PhI}(\mathrm{OAc})_{2}$ and ammonium carbamate or acetate. Addition of the sulfoxide resulted only in the formation of hypervalent iodonium salt 16. The identity of the iodonitrene $\left(\mathrm{PhI}=\mathrm{N}^{+}\right)$, was also confirmed via HRMS by labeling experiments using ${ }^{15} \mathrm{~N}$-labeled ammonium acetate. ${ }^{45}$

To summarize the mechanistic studies, it is likely that the N-source provides a sufficient concentration of ammonia which reacts with $\mathrm{PhI}(\mathrm{OAc})_{2}$ forming either iminoiodinane 18 or iodonitrene 19 that react with the sulfoxide (Scheme 15). Assuming the involvement of 18 (Scheme 15, path a), the formation of the sulfoximine may occur by direct attack of the sulfoxide at nitrogen with displacement of iodobenzene, ${ }^{53}$ or attack at the iodine center with subsequent reductive elimination leading to the sulfoximine. ${ }^{4}$ With iodonitrene 19 (path b), the direct nucleophilic attack of the sulfur at the electrophilic nitrogen would directly furnish the iodonium salt according to NMR and MS observations. In both pathways, the nucleophilicity of the sulfoxide plays a role.

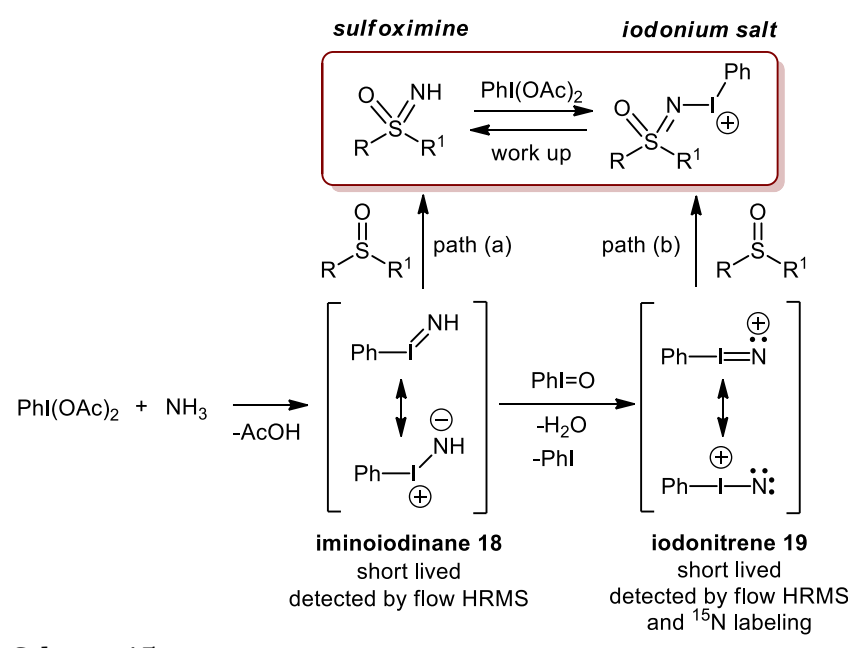

Scheme 15 
In the reaction forming sulfoximines from sulfides, 48 the mechanism for both $\mathrm{NH}$ and 0 transfer with the same reagents posed an even bigger question. Building on the on the data obtained in the reaction of sulfoxides a similar electrophilic nitrene or nitrenoid species would likely be involved. However, mechanistic differences, including the sequence of introduction of the heteroatoms, could be envisaged. Firstly, we observed that 2 equivalents of the oxidant were required. When less oxidant was used only the starting material and the product sulfoximine were present and intermediate species were not observed. This indicates that a more reactive intermediate species is formed. Therefore, we performed a series of experiments to establish whether $\mathrm{N}$ or $\mathrm{O}$ is transferred first. It is worth pointing out, that mechanistic aspects related to this process, have also been reported recently by Reboul and coworkers. ${ }^{55}$ In Scheme 16, a combination of the data from the chemical investigations reported by ourselves ${ }^{48}$ as well as by Reboul are presented.

b)

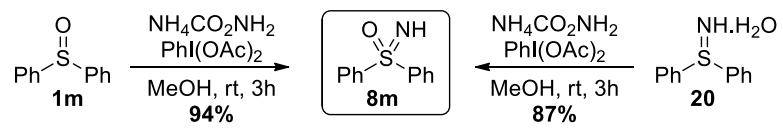

c)

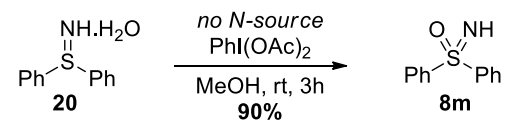

d)

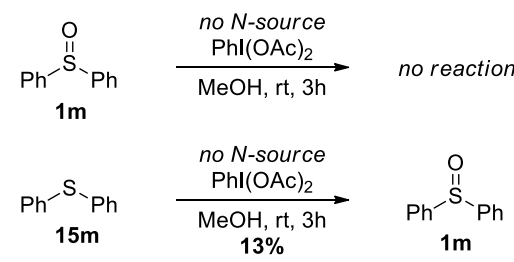

$$
\text { f) }
$$

conditions:

i): $\mathrm{Phl}(\mathrm{OAc})_{2}$ (3 equiv.), $\mathrm{NH}_{4} \mathrm{CO}_{2} \mathrm{NH}_{2}$ (2.5 equiv.), $\mathrm{MeOH}, \mathrm{rt}, 30 \mathrm{~min}$

ii): $\mathrm{Phl}(\mathrm{OAc})_{2}$ (3 equiv.), $\mathrm{NH}_{4} \mathrm{CO}_{2} \mathrm{NH}_{2}$ (2.5 equiv.), $\mathrm{CH}_{3} \mathrm{CN}, \mathrm{rt}, 2 \mathrm{~h}$

iii): $\mathrm{Phl}(\mathrm{OAc})_{2}$ (3 equiv.), $\mathrm{NH}_{3}$ (5 equiv.), $\mathrm{CH}_{3} \mathrm{CN}, \mathrm{H}_{2} \mathrm{O}$ (10 equiv.), rt, $30 \mathrm{~min}$

\section{Scheme 16}

By using diphenylsulfide $\mathbf{1 5 m}$ in the presence of ammonia and $\mathrm{PhI}(\mathrm{OAc})_{2}$ in acetonitrile and water, diphenylsulfoximine $\mathbf{8 m}$ could be obtained in $78 \%$ yield (Scheme 22,a). Complete selectivity, for the formation of sulfoximine $\mathbf{8 m}$, was also observed starting either from diphenyl sulfoxide $\mathbf{1 m}$, or diphenylsulfilimine $\mathbf{2 1}$ (Scheme 16,b). From sulfilimine 20, reaction with $\mathrm{PhI}(\mathrm{OAc})_{2}$, in the absence of the $\mathrm{N}$-source, led to sulfoximine $\mathbf{8 m}$ resulting from an oxidation process (Scheme 16,c). However, either sulfoxide 1m, or sulfide $\mathbf{1 5} \mathbf{m}$ in the absence of the $\mathrm{N}$-source gave little or no reaction, which appears crucial to the excellent chemoselectivity of the processes (Scheme 16,d,e). Further useful insights were obtained studying the reactivity of benzylphenylsulfide $\mathbf{1 5 b}$. On reacting $\mathbf{1 5 b}$ with
PhI(OAc) $)_{2}$ a complex mixture was obtained, in striking contrasts with a $92 \%$ yield of sulfoximine $\mathbf{8 b}$ in the presence of ammonium carbamate (Scheme 16, f, conditions i). Interestingly, Reboul demonstrated that water could be playing a role in the reaction; in acetonitrile, no reaction occurred when water was removed from the reaction mixture (Scheme 16, f, conditions ii). However, it is not necessary to be the source of the sulfoximine oxygen. By contrast, in the presence of 10 equiv of water, using acetonitrile as reaction solvent a 1:1 mixture of sulfoximine $\mathbf{8 b}$ and the corresponding $\mathrm{N}$ acyl derivative $\mathbf{2 1}$ was observed (Scheme 16, f, conditions i). These results support the hypothesis that the nitrogen is likely transferred first, and that the corresponding sulfilimine is further oxidized to intermediates that are broken down by the methanol solvent or water.

Based on labeling experiments, HRMS and ${ }^{1} \mathrm{H},{ }^{13} \mathrm{C}$ and ${ }^{15} \mathrm{~N}$ NMR investigation, Reboul proposed the mechanism reported in Scheme 17.55 The previously proposed iodonitrene $\mathbf{1 9}$ is likely to be the Ndonor to the sulfur, 45,48 leading to a short lived sulfilimine iodonium species 22. Intermediate $\mathbf{2 2}$ is attacked by methoxy or acetate anion with formation of methoxy- or acetoxy- $\lambda^{6}$-sulfanenitrile $\mathbf{2 3}$ and $\mathbf{2 4}$ respectively. Interestingly, $\mathbf{2 3}$ and $\mathbf{2 4}$ have been characterized by HRMS, isotopic labeling and multinuclear magnetic resonance. 55

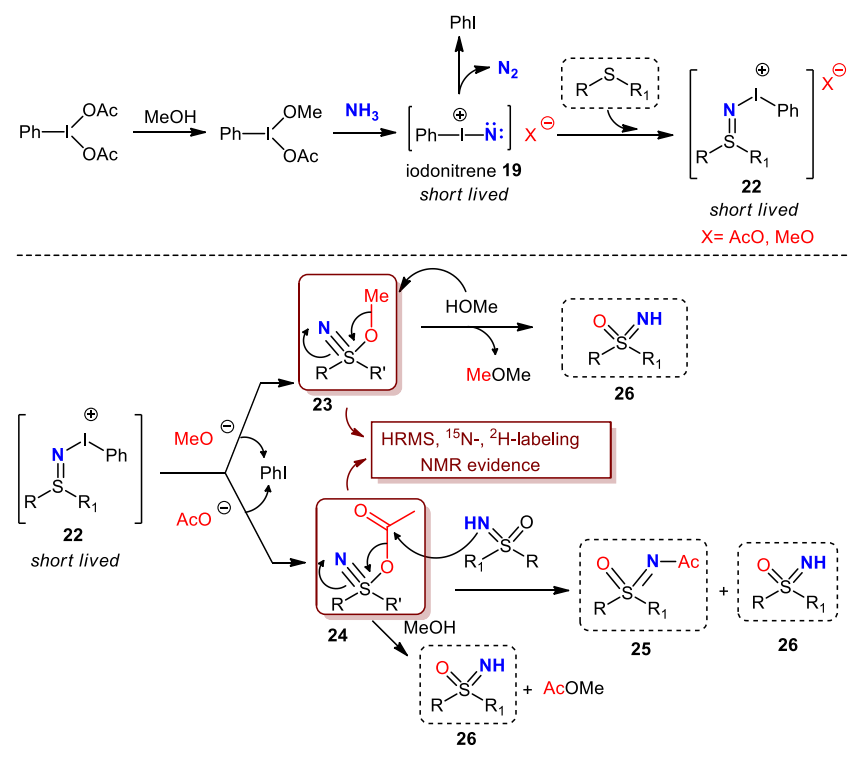

\section{Scheme 17}

Methoxy- $\lambda 6$-sulfanenitrile 23 could undergo to a nucleophilic attack of methanol with formation of dimethylether and the corresponding sulfoximine $\mathbf{2 6}$. Similarly, acetoxy- $\lambda^{6}$-sulfanenitrile 24 may behave as an acetylating agent either toward sulfoximine or methanol leading to $\mathbf{N}$-acyl-sulfoximine $\mathbf{2 5}$ and $\mathrm{NH}$-sulfoximine 26 (Scheme 17). This mechanism supports the hypothesis that the oxygen is derived from methanol or acetate.

\section{Further applications}

In this last section, we wish to report further interesting applications of the strategies herein described for the preparation of NH-sulfoximines. Firstly, the potential to use alternative Nsources, such as the cheap and easy to handle ammonium acetate, was demonstrated in the preparation of sulfoximines $(S)-\mathbf{8 a}, \mathbf{8 p}$ and the MSO precursor 12 in good yields (Scheme 18).45 


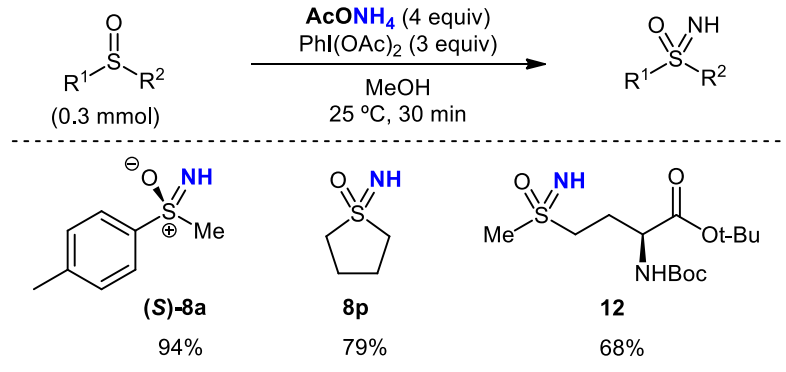

\section{Scheme 18}

The preparation of ${ }^{15} \mathrm{~N}$-labeled sulfoximines from biologically relevant sulfides was readily achieved, exploiting the functional group compatibility of the $\mathrm{NH}$ and O-transfer strategy. Using ${ }^{15} \mathrm{~N}-$ labeled ammonium acetate as the $\mathrm{N}$-source, the reaction with protected methionine, dipeptides, and also biotin afforded the corresponding ${ }^{15} \mathrm{NH}$-sulfoximines ${ }^{15} \mathbf{N}-\mathbf{2 7 - 3 0}$ in good yields as mixtures of diastereoisomers (Scheme 19). ${ }^{48}$

$$
\text { (15) }
$$

\section{Scheme19}

Lücking employed the Rh-catalyzed strategy for the preparation of sulfoximine carbamates, in the synthesis of sulfoximine analogues of palbociclib and ribociclib, which are selective CDK4/6 inhibitors used in cancer therapy. N-Boc sulfoximine $\mathbf{3 2}$ was the key intermediate in the preparation of analogues $\mathbf{3 3}$ and $\mathbf{3 4}$ obtained after facile removal of the Boc group (Scheme 20, a).14
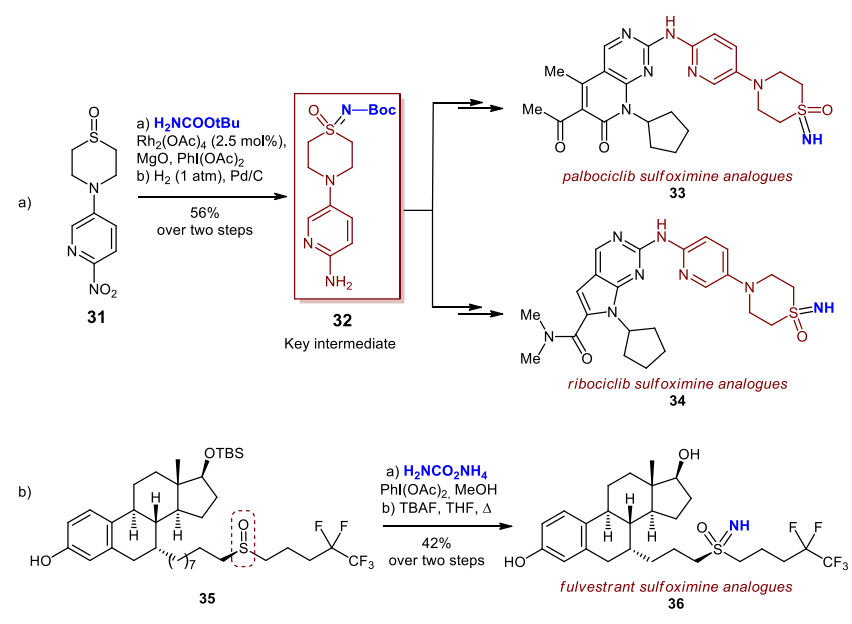

\section{Scheme 20}

The strategy for the direct NH-transfer to sulfoxide, has been applied by Lücking for the preparation of the sulfoximine analogues of fulvestrant, a selective estrogen receptor degrader
(SERD) that both antagonizes and degrades ER- $\alpha$ and is active in patients experiencing disease progression on antihormonal agents. The synthesis of analogues $\mathbf{3 6}$ was accomplished in two steps including a stereospecific $\mathrm{N}$-transfer with ammonium carbamate and $\mathrm{PhI}(\mathrm{OAc})_{2}$ followed by TBS deprotection (Scheme 20, b). ${ }^{14}$

\section{Conclusion}

In conclusion, in this account we collected our recent achievements in the development of straightforward strategies for accessing $\mathrm{NH}$ sulfoximines, and reviewed these in the context of the field. Both metal-based and metal-free approaches have been described as well as useful synthetic applications. The rhodium catalysed synthesis of sulfoximine carbamates allows the introduction of orthogonal and easily removable protecting groups into a molecule. From a sustainability point of view, the metal-free approach avoids the use of expensive transition metal catalysts and offers synthetic efficiency to free NH sulfoximines. The metal-free protocols are suitable for converting both sulfoxides and sulfides directly to sulfoximines, and appear to be widely applicable. In addition, the protocols tolerate functionality in the substrate, and are suitable for both late stage introduction of the sulfoximine moiety and for ${ }^{15} \mathrm{~N}$-labeling. From a mechanistic point of view, available spectroscopic and spectrometric measurements suggest a iodonitrene or iminoiodinane species as reactive and short-lived intermediates. It is expected that these strategies will lead to impacts in the near future in sulfoximine chemistry, drug discovery programs as well as in other $\mathrm{N}$-transfer reactions.

\section{Acknowledgment}

We gratefully acknowledge The Royal Society for a University Research Fellowship (to J. A. B.). The University of Bari for support.

\section{References}

(1) Bentley, H. R.; McDermott, E. E.; Pace, J.; Whitehead, J. K.; Moran, T. Nature 1949, 163, 675.

(2) Reggelin, M.; Zur, C. Synthesis, 2000, 1.

(3) a) Koep, S.; Gais, H.-J.; Raabe, G.; J. Am. Chem. Soc. 2003, 125, 13243. b) X. Shen, W. Miao, C. Ni, J. Hu, Angew. Chem. Int. Ed. 2014, 53, 775. c) Frings, M.; Atodiresei, I.; Wang, Y.; Runsink, J.; Raabe, G.; Bolm, C. Chem. Eur. J. 2010, 16, 4577. d) Shen, X.; Liu, Q.; Zhang, W.; Hu, J. Eur. J. Org. Chem. 2016, 906.

(4) a) Bolm, C.; Verrucci, M.; Simic, O.; Cozzi, P. G.; Raabe, G.; Okamura, H. Chem. Commun. 2003, 2826; b) Langner, M.; Bolm, C. Angew. Chem. Int. Ed. 2004, 43, 5984; c) Langner, M.; Remy, P.; Bolm, C. Chem. Eur. J. 2005, 11, 6254 .

(5) a) Yadav, M. R.; Rit, R. K.; Sahoo A. K. Org. Lett. 2013, 15, 1638. b) Yadav, M. R.; Rit, R. K.; Sahoo A. K. Chem. Eur. J. 2012, 18, 5541. c) Rit, R. K.; Yadav, M. R.; Ghosh, K.; Shankar, M.; Sahoo, A. K. Org. Lett. 2014, 16, 5258. d) Ghosh, K.; Rit, R. K.; Ramesh, E.; Sahoo, A. K. Angew. Chem. Int. Ed. 2016, 55, 7821. e) Cheng, Y.; Dong, W.; Parthasarathy, K.; Bolm, C. Org. Lett. 2017, 19, 726.

(6) Gnamm, C.; Jeanguenat, A.; Dutton, A. C.; Grimm, C.; Kloer, D. P.; Crossthwaite, A. J. Bioorg. Med. Chem. Lett. 2012, 22, 3800.

(7) Lücking, U. Angew. Chem. Int. Ed. 2013, 52, 9399.

(8) a) Bizet, V.; Hendriks, C. M. M.; Bolm, C. Chem. Soc. Rev., 2015, 44, 3378. b) Worch, C.; Mayer, A. C.; Bolm, C. In Organosulfur Chemistry in Asymmetric Synthesis; Toru, T., Bolm, C., Eds.; Wiley-VCH: Weinheim, 2008; $\mathrm{p} 209$.

(9) For the use of flow chemistry in sulfoximine synthesis, see: a) Lebel, H.; Piras, H.; Borduy, M. ACS Catal. 2016, 6, 1109. b) Gutmann, B.; 
Elsner, P.; O'Kearney-McMullan, A.; Goundry, W.; Roberge, D. M.; Kappe, C. O. Org. Process Res. Dev. 2015, 19, 1062.

(10) For the cross-coupling of sulfoximine containing building blocks, see: a) Steinkamp, A.-D.; Wiezorek, S.; Brosge, F.; Bolm, C. Org. Lett., 2016, 18, 5348. b) Sirvent, J. A.; Bierer, D.; Webster, R.; Lücking, U. Synthesis 2017, 49, 1024. c) Battula, S. R. K.; Rama Kishore Putta, V. P.; Subbareddy, G. V.; Chakravarthy, I. E.; Saravanan, V. Org. Biomol. Chem. 2017, 3742. d) Cho, G. Y.; Okamura, H.; Bolm, C. J. Org. Chem. 2005, 70, 2346. e) Tota, A.; Fanelli, F.; Falcicchio, A.; Luisi, R.; Degennaro, L. Chem. Heterocycl. Compd. 2017, 53, 322-328.

(11) a) Goldberg, F. W.; Kettle, J. G.; Xiong, J.; Lin, D. Tetrahedron 2014, 70, 6613. b) Goldberg, F. W.; Kettle, J. G.; Kogej, T.; Perry, M. W. D.; Tomkinson, N. P. Drug Discov. Today 2015, 20, 11.

(12) a) Lücking, U.; Jautelat, R.; Krüger, M.; Brumby, T.; Lienau, P.; Schäfer, M.; Briem, H.; Schulze, J.; Hillisch, A.; Reichel, A.; Wengner, A. M.; Siemeister, G. Chem. Med. Chem. 2013, 8, 1067. b) Siemeister, G.; Lucking, U.; Wengner, A. M.; Lienau, P.; Steinke, W.; Schatz, C.; Mumberg, D.; Ziegelbauer, K. Mol. Cancer Ther. 2012, 11, 2265. c) Foote, K. M.; Lau, A.; M Nissink, J. W. Future Med. Chem. 2015, 7, 873. d) Foote, K. M.; Nissink, J.W. M.; Turner, P. Morpholino pyrimidines and their use in therapy, AstraZeneca Patent W02011/154737 A1, 2011.

(13) Frings, M.; Bolm, C.; Blum, A.; Gnamm, C. Eur. J. Med. Chem. 2017, 126, 225.

(14) Sirvent, J. A.; Lücking, U. Chem. Med. Chem. 2017, 12, 487.

(15) Teng, F.; Cheng, J.; Bolm, C. Org. Lett. 2015, 17, 3166.

(16) Sedelmeier, J.; Bolm, C. J. Org. Chem. 2005, 70, 6904.

(17) a) Priebbenow, D. L.; Bolm, C. Org. Lett. 2014, 16, 1650. b) Muneeswara, M.; Kotha, S. S.; Sekar, G. Synthesis, 2016, 48, 1541.

(18) a) Chen, X. Y.; Wang, L.; Frings, M.; Bolm, C. Org. Lett. 2014, 16, 3796. b) Wang, H.; Cheng, Y.; Becker, P.; Raabe, G.; Bolm, C. Angew. Chem. Int. Ed. 2016, 55,12655.

(19) Cheng, Y.; Dong, W.; Wang, L.; Parthasarathy, K.; Bolm, C. Org. Lett. 2014, 16, 2000.

(20) Cheng, H.; Wen, J.; Bolm, C. Chem. A Eur. J. 2017, DOI: 10.1002/chem.201700953.

(21) Teng, F.; Yu, J. T.; Zhou, Z.; Chu, H.; Cheng, J. J. Org. Chem. 2015, 80 , 2822.

(22) Bohnen, C.; Bolm, C. Org. Lett. 2015, 17, 3011.

(23) Zhu, H.; Yu, J.-T.; Cheng, J. Chem. Commun., 2016, 52, 11908.

(24) Wang, H.; Frings, M.; Bolm, C. Org. Lett. 2016, 18, 2431.

(25) Dong, S.; Frings, M.; Cheng, H.; Wen, J.; Zhang, D.; Raabe, G.; Bolm, C. J. Am. Chem. Soc. 2016, 138, 2166.

(26) a) Tomooka, C. S.; Carreira, E. M. Helv. Chim. Acta, 2002, 3773. b) Mancheno, O. G.; Bolm, C. Chem. Eur. J. 2007, 13, 6674. c) Lebel, H.; Piras, H.; Bartholoméüs, J. Angew. Chem. Int. Ed. 2014, 53, 7300.

(27) a) Wang, J.; Frings, M.; Bolm, C. Chem. A Eur. J. 2014, 20, 966. b) Müller, J. F. K.; Vogt, P. Tetrahedron Lett. 1998, 39, 4805. c) Cren, S.; Kinahan, T. C.; Skinner, C. L.; Tye, H. Tetrahedron Lett. 2002, 43, 2749. d) Lacôte, E.; Amatore, M.; Fensterbank, L.; Malacria, M. Synlett 2002, 116. e) Cho, G. Y.; Bolm, C. Org. Lett. 2005, 7, 4983.

(28) Okamura, H.; Bolm, C. Org. Lett. 2004, 6, 1305.

(29) a) Bizet, V.; Buglioni, L.; Bolm, C. Angew. Chem. Int. Ed. 2014, 53, 5639. b) Bizet, V.; Bolm, C. Eur. J. Org. Chem. 2015, 2015, 2854.
(30) Miao, J.; Richards, N. G. J.; Ge, H. Chem. Commun. 2014, 50, 9687.

(31) a) Bach, T.; Körber, C. Tetrahedron Lett. 1998, 39, 5015. b) Bach, T.; Körber, C. Eur. J. Org. Chem. 1999, 1033.

(32) Zenzola, M.; Doran, R.; Luisi, R.; Bull, J. A. J. Org. Chem. 2015, 80, 6391

(33) Johnson, C. R.; Kirchhoff, R. A.; Corkins, H. G. J. Org. Chem. 1974, 39, 2458.

(34) For related studies, see: Buglioni, L.; Bizet, V.; Bolm, C. Adv. Synth. Catal. 2014, 356, 2209. Also see: Marzag, H.; Schuler, M.; Tatibouët, A.; Reboul, V. Eur. J. Org. Chem. 2017, 2017, 896.

(35) Johnson, C. R.; Haake, M.; Schroeck, C. W. J. Am. Chem. Soc. 1970, 92, 6594.

(36) Wang, J.; Zhang, J.; Miao, K.; Yun, H.; Shen, H. C.; Zhao, W.; Liang C. Tetrahedron Lett. 2017, 58, 333.

(37) a) Mendiola, J.; Rincon, J. A.; Mateos, C.; Soriano, J. F.; de Frutos, O.; Niemeier, J. K.; Davis, E. M. Org. Process Res. Dev. 2009, 13, 263. b) Tamura, Y.; Minamikawa, J.; Sumoto, K.; Fujii, S.; Ikeda, M. J. Org. Chem. 1973, 38, 1239.

(38) Cho, G. Y.; Bolm, C. Tetrahedron Lett. 2005, 46, 8007.

(39) Dannenberg, C. A.; Fritze, L.; Krauskopf, F.; Bolm C. Org. Biomol. Chem., 2017,15, 1086.

(40) Krasnova, L. B.; Hili, R. M.; Chernoloz, O. V.; Yudin A. K. Arkivoc, 2005, $4,26$.

(41) Siu, T.; Yudin A. K. Org. Lett., 2002, 4, 1839.

(42) Mancheno, O. M.; Bolm, C. Org. Lett., 2007, 9, 3809.

(43) Stoss, P.; Satzinger, G. Tetrahedron Lett. 1973, 14, 267.

(44) Zenzola, M.; Doran, R.; Degennaro, L.; Luisi, R.; Bull, J. A. Angew. Chem. Int. Ed. 2016, 51, 7203.

(45) Espino, C. G.; Du Bois, J. Angew. Chem. Int. Ed. 2001, 40, 598-600.

(46) https://www.youtube.com/watch?v=4KpDQnGHi28.

(47) a) Collins, K. D.; Glorius, F. Nat. Chem. 2013, 5, 597. b) Collins, K. D.; Rühling, A.; Glorius, F. Nat. Protoc. 2014, 9, 1348. c) Collins, K. D.; Glorius, F. Acc. Chem. Res. 2015, 48, 619.

(48) Tota, A.; Zenzola, M.; Chawner, S. J.; St John-Campbell, S.; Carlucci, C.; Romanazzi, G.; Degennaro, L.; Bull, J. A.; Luisi, R. Chem. Commun. 2017, 53, 348 .

(49) Silva, L. F.; Lopes, N. P. Tetrahedron Lett. 2005, 46, 6023.

(50) Unpublished results.

(51) Iacobucci, C.; Reale, S.; De Angelis, F. Angew. Chem. Int. Ed. 2016, 55, 2980.

(52) Ivanov, A. S.; Popov, I. A.; Boldyrev, A. I.; Zhdankin, V. V. Angew. Chem. Int. Ed. 2014, 53, 9617.

(53) Ochiai, M.; Kaneaki, T.; Tada, N.; Miyamoto, K.; Chuman, H.; Shiro, M.; Hayashi, S.; Nakanishi, W. J. Am. Chem. Soc. 2007, 129, 12938.

(54) Dohi, T.; Kita, Y. Hypervalent Iodine, in Iodine Chemistry and Applications Ch. 7, pp103-157 (John Wiley \& Sons, Hoboken, NJ, 2015).

(55) Lohier, J.-F.; Glachet, T.; Marzag, H.; Gaumont, A.-C.; Reboul, V. Chem. Commun., 2017, 53, 2064. 


\section{Biosketches}

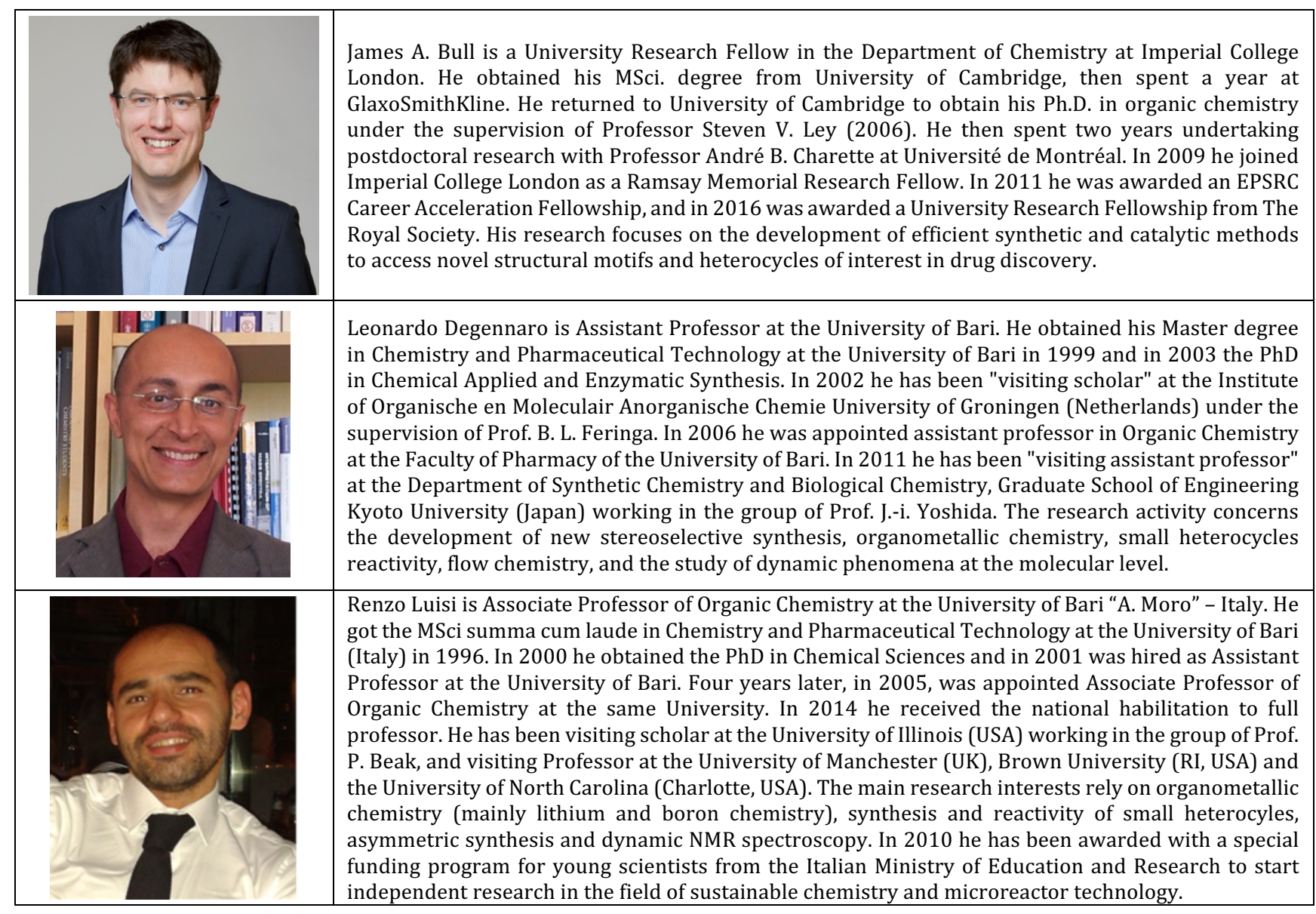

